\title{
Revisiting the welfare effects of eliminating business cycles
}

\author{
Per Krusell a,b,c,d, Toshihiko Mukoyama ${ }^{\mathrm{e}, \mathrm{f}}$, Ayşegül Şahing ${ }^{\mathrm{g}}$, Anthony A. Smith Jr. ${ }^{\mathrm{h}, *}$ \\ a Princeton University, United States \\ b IIES, Sweden \\ c CEPR, United Kingdom \\ d NBER, United States \\ e University of Virginia, United States \\ f CIREQ Canada \\ g Federal Reserve Bank of New York, United States \\ h Yale University, United States
}

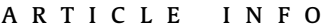

\section{Article history:}

Received 29 December 2008

Revised 1 January 2009

Available online 23 January 2009

\section{JEL classification:}

C68

D31

D61

E32

\section{Keywords:}

Cost of business cycles

Incomplete markets

Heterogeneity

\begin{abstract}
A B S T R A C T
We investigate the welfare effects of eliminating business cycles in a model with substantial consumer heterogeneity. The heterogeneity arises from uninsurable and idiosyncratic uncertainty in preferences and employment status. We calibrate the model to match the distribution of wealth in U.S. data and features of transitions between employment and unemployment. In comparison with much of the literature, we find rather large effects. For our benchmark model, we find welfare effects that, on average across all consumers, are of a bit more than one order of magnitude larger than those computed by Lucas [Lucas Jr., R.E., 1987. Models of Business Cycles. Basil Blackwell, New York]. When we distinguish long- from short-term unemployment, long-term unemployment being distinguished by poor (and highly procyclical) employment prospects and low unemployment compensation, the average gain from eliminating cycles is as much as $1 \%$ in consumption equivalents. In addition, in both models, there are large differences across groups: very poor consumers gain a lot when cycles are removed (the long-term unemployed as much as around 30\%), as do very rich consumers, whereas the majority of consumers-the "middle class"-sees much smaller gains from removing cycles. Inequality also rises substantially upon removing cycles.
\end{abstract}

(C) 2009 Elsevier Inc. All rights reserved.

\section{Introduction}

Lucas (1987) suggested a way of calculating an upper bound for the welfare gain associated with the elimination of business cycles. His calculation resulted in a very, very small number. If one accepts his analysis, indeed it is a major puzzle why so much effort is aimed at discussing stabilization policy, be it monetary or fiscal. Significant amounts of research have been generated during the 20 years since Lucas wrote his paper in an aim to justify an interest in stabilization. In a paper published in the Review of Economic Dynamics in 1999 by a subset of the authors of the present paper (Krusell and Smith),

\footnotetext{
(3) This paper is the complete version of the working paper by Krusell and Smith [Krusell, P., Smith Jr., A.A., 2002. Revisiting the welfare effects of eliminating business cycles. Manuscript. Yale University], which is a corrected and extended version of Krusell and Smith [Krusell, P., Smith Jr., A.A., 1999. On the welfare effects of eliminating business cycles. Review of Economic Dynamics 2, 245-272]. The views expressed in this article are those of the authors and do not necessarily reflect the position of the Federal Reserve Bank of New York or the Federal Reserve System. Krusell and Smith thank the National Science Foundation for support.

* Corresponding author at: Yale University, Economics, 28 Hillhouse Avenue, Room 306, New Haven, CT, USA.

E-mail address: tony.smith@yale.edu (A.A. Smith Jr.).
} 
we argued that one possible motivation for an interest in the welfare effects of business cycles is an asymmetric effect of cycles on different groups of consumers. The idea we put forth is that although cycles do not affect the "average household" much at all in welfare terms-Lucas considered a representative consumer in his calculation-some consumers may suffer significantly, especially the poor and the unemployed. We considered a setting with significant and, in important respects, realistic consumer heterogeneity and assessed the welfare effects of removing cycles for all subgroups of the population. Our results suggested larger, but still very small effects. However, in our 1999 analysis we made an assumption-inadvertentlythat, we have realized, is of great quantitative consequence. The goal of the present paper is to revisit our 1999 model and report the results of the much more appropriate assumption. The result is far greater scope for business cycles to generate welfare costs, especially for the poor and the unemployed.

Lucas did not address how aggregate cycles could be removed; his calculation was based on simply replacing, at no cost to society (hence the upper-bound nature of the results), an estimate of the aggregate consumption process with its mean. In our work, which uses a dynamic general-equilibrium model based on the stochastic growth model with idiosyncratic consumer productivity shocks and incomplete insurance against these shocks, we replace the aggregate productivity process, which is exogenous, with its mean, thus implementing the spirit of Lucas's approach. However, when aggregate shocks are removed, what might the implications be for individuals' shocks? The assumption adopted here turns out to be key, and the central purpose of the present paper is to argue for and explore a more appropriate assumption than the one we used in our earlier paper. There, our aim was to again stay as close in spirit as possible to Lucas's approach: we argued that the idiosyncratic shock process should be replaced, when aggregate cycles are removed, by one that "integrates out" the aggregate component of the individual shock. However, we implemented our integration principle incorrectly and thereby instead, in effect, made the assumption that individual shocks were unaffected by the removal of cycles. In the present paper, we instead show that a correct implementation of this principle implies that when the aggregate component is integrated out, the individual income process becomes less risky, thus by itself representing a (direct) welfare improvement. In addition, the lower amount of individual risk induces changes in equilibrium prices which also have important indirect effects on welfare.

By integrating the aggregate risk out of an income process we mean averaging over the aggregate states conditional on each idiosyncratic state. However, since individual employment-the source of income fluctuations for individuals in our model-is correlated with the aggregate state (the employment rate is procyclical), the individual (employment) variable is not fully idiosyncratic, which makes integration a nontrivial task. With correlation, one thus first needs to construct a pure process for idiosyncratic luck that, by definition, is uncorrelated across individuals. Conditional on every realization of this new idiosyncratic variable, one can then integrate out the influence of aggregate risk on individual employment. Following this procedure, we now find that the removal of aggregate risk lowers individual employment risk by about $16 \%$ in the long run in our baseline model.

The effect on individual risk has two implications. One is quantitative: it turns out to generate much larger effects on welfare, on average a bit more than one magnitude larger than those Lucas found. But there is also a qualitative effect regarding who gains and who loses from removing cycles: the effect is U-shaped in wealth. First, with less idiosyncratic risk, the poorest consumers gain significantly since they suffer most from risk; quantitatively, they gain up to several percentage points in consumption equivalents from eliminating cycles, thus contrasting Lucas's numbers, which are orders of magnitude lower. Second, due to the lower income risk, the amount of precautionary saving in the economy falls. In the closed economy that we study, this raises the interest rate. This effect is small but nevertheless significantly raises the welfare of the very richest, who own very large amounts of wealth; the wealth distribution in the initial state reproduces the observed Gini coefficient for wealth and thus has (a small number of) very wealthy individuals. This effect on the welfare of the very richest also amounts to several percentage points of consumption equivalents. The middle class, in contrast, sees an improvement because of the lowering of risk, but it is very well insured in utility terms, so this effect is almost nil. Moreover, the middle class, of which a typical agent is employed, sees a fall in the wage, and as a result the middle class-in total around $65 \%$ of the agents-experiences a welfare loss from eliminating aggregate cycles.

In addition to calculating welfare costs across individuals who differ in their economic status at the moment of eliminating cycles, we now obtain striking implications for long-run inequality. The Gini coefficient for wealth distribution in the steady state without aggregate cycles is over 0.9 , which is substantially larger than the initial average Gini coefficient of about 0.8 in the economy with cycles. Behind this result is the assumption that discount rates differ across consumers: as there is less risk, consumers with different discount rates tend to corners, thus making wealth more dispersed. In particular the poorest can afford to become even poorer, given that their income risk is less severe and their discount rates tend to be significantly above the interest rate: they "want to" become poorer. For example, the number of households with negative assets goes from 10 to $29 \%$. On the other hand, the very richest become even richer, due to the increase in interest rates.

An extension of our model distinguishes between short- and long-term unemployment: in essence, we assume here that once unemployed long enough, the probability of reentrance into employment falls significantly. In this case, we find that the removal of aggregate risk lowers individual employment risk by about $37 \%$ in the long run. Moreover, the gain from eliminating business cycles is much larger than in the model with only one kind of unemployment. Now everyone realizes a positive gain from eliminating business cycles; the average gain across the population, taking into account the transition to a new steady state, is as large as $1 \%$. The gains are very large for the consumers with very little wealth and very high wealth. In the long run, the wealth dispersion becomes very large. In particular, the fraction of agents with negative assets becomes $76 \%$. 
Because we are using the exact same model here as in the original paper, we describe it very compactly and move directly to the new analysis. Section 2 discusses the methodology and model used for answering our main question and Section 3 describes parameter choice and numerical issues. The main results are contained in Section 4 and the conclusions in Section 5; several appendices contain details on computation and other technical aspects of the analysis. ${ }^{1}$

\section{Preliminaries}

In Section 2.1, we first briefly discuss different routes one might take in answering our main question. We then discuss our theoretical model framework in Section 2.2 and, in Section 2.3, discuss in detail how we eliminate cycles.

\subsection{Methodology}

Lucas's (1987) model economy is very simple: consumption is exogenous and there is only one shock-one to the aggregate consumption process. In this economy, Lucas views the elimination of cycles as simply setting the shock to zero (its unconditional mean). To allow for heterogeneity, one way to extend what Lucas did is to use data on individual consumption. In particular, postulate and estimate the dependence of individual consumption on a purely idiosyncratic component and on aggregate variables. Next, for any value of the idiosyncratic component, take the average across the aggregate variables: this delivers a new consumption process. Finally, evaluate individual utility given this new process. We did not follow this procedure for two reasons. First, the procedure requires a long enough panel of individual consumption data so that one can reliably estimate a process for individual consumption which not only identifies the aggregate from the idiosyncratic component but also delivers an accurate assessment of the serial correlation properties of the shocks to individual consumption. Existing data do not grant this possibility. Second, this kind of calculation tends to underestimate the costs of cycles: if one instead models the randomness the agents are subject to, it may be better for them to change consumption in some other way than just averaging it across the aggregate states. That is, the utility value of not having aggregate cycles is underestimated. Although this is a problem also in Lucas's analysis, it is likely to be quantitatively more important here, where individual consumption volatility is much higher than in a representative-agent setup calibrated to aggregate data.

An alternative is to use individual income-for which data are arguably more reliable-and employ a model to infer consumption by assuming rational behavior given a certain set of asset markets. A first step would be to estimate a wage process and a process for asset returns and then to compare the utility outcome for a rational agent facing these processes with one where the same agent faces the same processes with their aggregate components removed. A second step would be to add an equilibrium component to the analysis, i.e., to also model where wages and rental rates come from. Since savings are likely to change as a result of eliminating risk, this seems a potentially important channel not to forget, at least if one believes that the economy is closed. Moreover, labor supply could change, leading to changes in the wage rate.

In this paper we follow a simple version of the latter procedure: we only model idiosyncratic differences in employment (and not in wage per hour worked), we assume that labor supply is inelastic, and we assume that all agents face the same return on saving. The asset structure is simple: there is only one asset-aggregate capital-and an exogenous borrowing constraint. The aggregate shock is modeled as exogenous changes in aggregate productivity and labor demand, and we study the general equilibrium effects for different groups of consumers by replacing the latter shocks with their conditional means.

\subsection{Model setup}

Except in the details of the individual's income process, the model is that in Krusell and Smith (1998); thus, we describe it compactly and, for the most part, without comment.

Consumers are ex-ante identical, with preferences

$$
E_{0} \sum_{t=0}^{\infty} \beta_{t} U\left(c_{t}\right),
$$

where $U(c)=\log (c)$ and $\beta_{t}$ is a stochastic variable which is idiosyncratic-i.i.d. across agents-and describes the cumulative discounting between period 0 and period $t$. In particular, $\beta_{t+1}=\tilde{\beta} \beta_{t}$, where $\tilde{\beta}$ is a three-state, first-order Markov process.

The aggregate production function is

$$
z \bar{k}^{\alpha} \bar{n}^{1-\alpha},
$$

where $k$ is capital (a bar refers to a total) and $n$ is labor, and $z$ is the aggregate productivity level. There is also home production, which accrues in the amount $\psi$ to all unemployed agents. ${ }^{2}$ Aggregate output, including undepreciated capital, can be used to either consume or invest.

\footnotetext{
1 The appendices are available upon request from the authors and can also be found at http://www.economicdynamics.org/RED15.htm.

2 Throughout the paper, we interpret "home production" as unemployment insurance, thereby avoiding, for simplicity, the explicit modeling of a government budget constraint. In the model which distinguishes short- from long-term unemployment, $\psi$ depends on whether an unemployed consumer is in the short- or long-term unemployed state.
} 
In the model with homogeneous unemployment, we denote the employment status $\epsilon=0$ for unemployment and $\epsilon=1$ for employment. When we distinguish short- and long-term unemployment, we introduce a new notation: $\epsilon=l$ for longterm unemployment, $\epsilon=s$ for short-term unemployment, and $\epsilon=e$ for employment. The distinction between short- and long-term unemployment allows us to consider differences among the unemployed both in terms of their income when unemployed and their prospects for future employment. In particular, in the calibration we assume (i) that the short-term unemployed receive higher unemployment insurance benefits (for the unemployment benefit $\psi_{\epsilon}, \psi_{s}>\psi_{l}>\psi_{e}=0$ holds); and (ii) that their probability of employment is higher, with the difference being more pronounced in recessions than in booms. As before, the individual employment status, jointly with the aggregate shock $z$, follows a first-order Markov process

Formally, a recursive competitive equilibrium for this economy is defined using the aggregate state variables. Let $\Gamma$ denote the current measure of consumers over holdings of capital, employment, and preference status. Then, the state variable relevant to the individual includes $(\Gamma, z)$ and the idiosyncratic vector $(k, \epsilon, \tilde{\beta})$. Let $H$ denote the equilibrium transition function for $\Gamma$ :

$$
\Gamma^{\prime}=H\left(\Gamma, z, z^{\prime}\right) .
$$

Consumers solve

$$
v(k, \epsilon, \tilde{\beta} ; \Gamma, z)=\max _{c, k^{\prime}}\left\{U(c)+\tilde{\beta} E\left[v\left(k^{\prime}, \epsilon^{\prime}, \tilde{\beta}^{\prime} ; \Gamma^{\prime}, z^{\prime}\right) \mid z, \epsilon, \tilde{\beta}\right]\right\}
$$

subject to:

$$
\begin{aligned}
& c+k^{\prime}=r\left(\bar{k}, 1-u_{z}, z\right) k+w\left(\bar{k}, 1-u_{z}, z\right) I_{\epsilon=e}+\psi_{\epsilon}+(1-\delta) k^{\prime}, \\
& \Gamma^{\prime}=H\left(\Gamma, z, z^{\prime}\right), \\
& k^{\prime} \geqslant \underline{k},
\end{aligned}
$$

where $I_{\epsilon=e}=1$ if $\epsilon=e$ (or $\epsilon=1$ in the homogeneous unemployment case) and 0 otherwise, $r$ is the rental rate of capital, $w$ is the wage rate, $\delta$ is the depreciation rate, and $u_{z}$ is the unemployment rate when aggregate state is $z$. $\underline{k}$ is the exogenously set borrowing constraint. If

$$
k^{\prime}=f(k, \epsilon, \tilde{\beta} ; \Gamma, z)
$$

denotes the optimal saving decision for the agent, then an equilibrium can be defined as a law of motion $H$, individual functions $(v, f)$, and pricing functions $(r, w)$ such that (i) $(v, f)$ solves the consumer's problem; (ii) $(r, w)$ equal the marginal products of capital and labor, respectively; and (iii) $H$ is generated by $f$ and the law of motion for $(z, \epsilon, \tilde{\beta})$.

Finally, the economy without cycles is defined in the same way, but using different processes for $z$ (which is now deterministic) and $\epsilon$. We will now describe in detail how these processes are obtained.

\subsection{The elimination of cycles}

The heterogeneous-agent model we use here allows an indirect way-given the assumptions underlying the modelof deducing how individuals' consumption processes depend on aggregates. Ideally, one would then perhaps introduce stabilization policy explicitly in the model and compute the implied welfare effects. In our model, however, $z$ and $\epsilon$, which are exogenous processes, would then be left unchanged, and it is not clear that such an experiment would capture all potential gains from stabilization. Instead, and with the kind of "upper-bound approach" Lucas used, we opt for changing these processes directly, and more specifically changing them by removing any cyclical components, without introducing policy explicitly.

How should the processes be altered, i.e., how should the cyclical components be removed? We discuss this next.

\subsubsection{Aggregates}

With reference to Lucas's procedure, which involved replacing the consumption shock in his model with its mean, assuming that there could be no average consumption gain-or loss-from eliminating cycles, we also adopt a "neutrality" assumption. For aggregate shocks, we replace $z$ and $u$ by their conditional means. In the long run, our economy without cycles thus has productivity $\pi_{g} z_{g}+\pi_{b} z_{b}$ and unemployment rate $\pi_{g} u_{g}+\pi_{b} u_{b}$, with $\pi_{g}$ and $\pi_{b}$ representing the unconditional probabilities of good and bad aggregate states, respectively.

\subsubsection{Individual variables}

One could remove the aggregate component of the individual shock in several ways. We proposed, in our 1999 paper, to integrate over the aggregate shock. Suppose the individual variable of interest, $y$, is a function $g$ of two independent random variables, $y=h(i, z)$, where $i$ is an idiosyncratic shock and $z$ is an aggregate shock. The assumption that the two shocks are independent amounts to a definition of "idiosyncratic"; the densities are denoted $f_{i}(i)$ and $f_{z}(z)$, respectively. We then identify the idiosyncratic shock process in the absence of aggregate risk, $y^{w / o}(i)$, with the following:

$$
y^{w / o}(i)=\int_{z} h(i, z) f_{z}(z) d z
$$


for each $i$, with density $f_{i}(i)$.

For the most straightforward example, suppose that $y$ denotes an individual productivity level and that this productivity is the sum of two jointly normal shocks, one individual-specific, but not necessarily fully idiosyncratic, shock $\epsilon$ and the aggregate shock $z$ :

$$
y=\epsilon+z .
$$

We assume first that the marginal distribution of each of these shocks is $N(0,1)$ and that the covariance between the two shocks is $\rho$. If $\rho=0$, so that $\epsilon$ and $z$ are independent, we deduce that $y$ is $N(0,2)$. Then we obtain, using our integration principle, that

$$
y^{w / 0}=\epsilon,
$$

which is $N(0,1)$. Here, $y^{w / 0}$ is clearly less risky-it has a lower variance than $y .^{3}$

If $\epsilon$ and $z$ are correlated, integration requires first projecting $y$ onto $z$. This projection is $(1+\rho) z+i$, where $i$ and $z$ are now independent by construction and $i$ has variance $1-\rho^{2}$, since we assumed that both $\epsilon$ and $z$ were $N(0,1)$. Note that $y$ in this case is distributed according to $N(0,2+2 \rho)$. Now integration implies that

$$
y^{w / 0}=i,
$$

which is $N\left(0,1-\rho^{2}\right)$ : this process always has a lower variance than $y$, and this variance vanishes entirely if the individual and aggregate components that comprise $y$ are perfectly correlated.

In our model framework, the individual-specific income process $y$ depends crucially on the employment process $\epsilon$, which is not, in general, independent of $z$, like in the last example. In order to find the $\epsilon^{w / 0}$-the employment process when there are no aggregate shocks-we therefore need to do the equivalent of the linear projection that was appropriate in that last example: we need to design a purely idiosyncratic variable $i$ such that the employment/income outcome $\epsilon$ is a function of $i$ and $z .{ }^{4}$ It turns out that this can be done as follows: let $i$ be uniform on $[0,1]$, and define $\epsilon\left(i, z_{g}\right)$ to be 1 if $i \leqslant \pi_{1 \mid g}$ and 0 otherwise and $\epsilon\left(i, z_{b}\right)$ to be 1 if $i \leqslant \pi_{1 \mid b}$ and 0 otherwise, where $\pi_{1 \mid z}$ is the probability of being employed when the aggregate state is $z$. We will assume in this discussion that $\pi_{1 \mid g}>\pi_{1 \mid b}$, i.e., that $\epsilon$ and $z$ are positively correlated.

Integration is now straightforward. If $i \leqslant \pi_{1 \mid b}$, the individual is employed no matter what happens to the aggregate shock, so the integration is trivial: $\epsilon^{w / 0}=1$ for such values of $i$. Similarly, if $i>\pi_{1 \mid g}$, the individual is unemployed no matter what: $\epsilon^{w / o}=0$. Finally, if $\pi_{1 \mid b}<i<\pi_{1 \mid g}$, the individual is employed only if the aggregate state is good, which occurs with probability $\pi_{g}$; thus, integration for such values of $i$ implies that $\epsilon^{w / 0}=\pi_{g} \cdot 1+\left(1-\pi_{g}\right) \cdot 0=\pi_{g}$. Thus, our new employment variable $\epsilon^{W / o}$ has the following 3-state distribution: 1 with probability $\pi_{1 \mid b}, \pi_{g}$ with probability $\pi_{1 \mid g}-\pi_{1 \mid b}$, and 0 with probability $1-\pi_{1 \mid g}$. Note that the new income variable thus has a different support-one more state-and that it is less risky: some probability mass has been moved from the extremes 0 and 1 into a middle state. In a dynamic economy, where individual employment is correlated over time, one can follow the same principles but it is quite a nontrivial affair to find the process for $\epsilon^{w / 0}$. Suffice it to say here that this new process (i) will change nature-it will increase its support-as time evolves; (ii) will not be first-order Markov, but rather will be a function of two state variables (in the homogeneous unemployment case) which in turn are a function of all present and past values of $i$ and evolve recursively; and (iii) will settle down to a stationary process with full support on $[0,1]$. With both short- and long-term unemployment, the number of state variables becomes five. Section 4 and Appendices A and B outline all the details for the baseline case; Appendix D gives the details for the case with two kinds of unemployment.

As stated above, has the integration principle been used in the existing literature? İmrohoroğlu (1989) did something in this direction but she restricted the new employment process to be first-order Markov, something which is inconsistent with our integration principle. Similarly, the procedure used in Storesletten et al. (2001) is also inconsistent with the integration principle, although both these authors and İmrohoroğlu propose individual processes in their economies without aggregate cycles that have some intuitive appeal. Finally, Krebs $(2003,2006)$ and Mukoyama and Şahin $(2006,2005)$ do adhere to the integration principle in their recent papers. ${ }^{5}$

\section{Parameter selection and model solution}

We now turn to the quantitative experiment, starting with the calibration and then briefly commenting on computation.

\footnotetext{
${ }^{3}$ Atkeson and Phelan (1994) assume that when the aggregate shock is removed, the individual shocks remain the same, but they become independent: they no longer move together. Thus, it is clear that the integration principle we propose is different.

${ }^{4}$ Krusell and Smith (1999) stated the integration principle as we did here (although with somewhat less detail) but failed to apply it correctly. The mistake amounted to treating $\epsilon$ as independent of $z$ in the actual application. As a result the exact same employment process was applied for the models with and without aggregate shocks. Thus, our results coincided with those that would follow from using Atkeson and Phelan's procedure: no risk was removed when cycles were removed.

${ }^{5}$ However, these papers appeared after the first working paper version of the present paper, i.e., Krusell and Smith (2002).
} 


\subsection{Calibration}

Our calibration here is identical to that in Krusell and Smith (1999); we therefore describe it only very briefly. The calibration is quite standard and mostly based on Krusell and Smith (1998); the main exception is the employment process, which in our alternative calibration now distinguishes short- from long-term unemployment.

We set, with a period being one quarter, $\delta=0.025$ and $\alpha=0.36$, and we use discount factors (for the benchmark model, set primarily in order to match the wealth distribution) that are $0.9858,0.9894$, and 0.9930 , with $80 \%$ of the population on the middle value and $10 \%$ on each extreme point in any time period and an expected duration of the extreme discount values of 50 years (approximating a lifetime); transitions can only occur to adjacent values. We let $u_{b}$ equal $10 \%$ and $u_{g}$ equal $4 \%$, and we set $z_{g}=1.01$ and $z_{b}=0.99$, with an expected duration of each aggregate state to 2 years. We set the borrowing constraint to be very loose, with a limit of about $60-70 \%$ of average annual income.

The first employment process we consider has the short- and long-term unemployment states collapsed into one state, as in Krusell and Smith (1998) exactly; this is our baseline calibration. It has $\psi_{0}=0.0334$, which is about $10 \%$ of the quarterly wage. The employment process here is given by four 2-by-2 matrices, one for each $\left(z, z^{\prime}\right)$ :

$$
\left(\begin{array}{ll}
0.33 & 0.67 \\
0.03 & 0.97
\end{array}\right)
$$

for the transition $\left(z, z^{\prime}\right)=\left(z_{g}, z_{g}\right)$ (rows/columns stand for the current/next period's state; row $1 / 2$ is the unemployment/employment state),

$$
\left(\begin{array}{ll}
0.75 & 0.25 \\
0.07 & 0.93
\end{array}\right)
$$

for $\left(z_{g}, z_{b}\right)$,

$$
\left(\begin{array}{ll}
0.25 & 0.75 \\
0.02 & 0.98
\end{array}\right)
$$

for $\left(z_{b}, z_{g}\right)$, and

$$
\left(\begin{array}{ll}
0.60 & 0.40 \\
0.04 & 0.96
\end{array}\right)
$$

for $\left(z_{b}, z_{b}\right)$. These parameters imply that the aggregate unemployment only take on two values and that the expected duration of unemployment is 1.5/2.5 quarters conditional on the good/bad aggregate state remaining. Because unemployment is higher in bad times than in good times, income risk due to unemployment risk is countercyclical, and integrating out the aggregate component from the employment dynamics will, as we shall see, lower the risk that the worker faces.

The second calibration uses the discount factors $0.9823,0.9879$, and 0.9935 , along with $\psi_{s}=0.391$, which is about $50 \%$ of the quarterly wage, to roughly replicate the U.S. replacement ratio during the first quarter of unemployment; $\psi_{l}=0.038$, which helps us match the left tail of the wealth distribution. The employment transition matrices here are:

$$
\left(\begin{array}{ccc}
0.50 & 0 & 0.50 \\
0.25 & 0 & 0.75 \\
0 & 0.03 & 0.97
\end{array}\right)
$$

for the $\left(z_{g}, z_{g}\right)$ transition (row/column 1 means long-term unemployment, 2 means short-term unemployment, and 3 means employment),

$$
\left(\begin{array}{ccc}
0.17 & 0 & 0.83 \\
0.03 & 0 & 0.97 \\
0 & 0.03 & 0.97
\end{array}\right)
$$

for the $\left(z_{b}, z_{g}\right)$ transition,

$$
\left(\begin{array}{ccc}
0.94 & 0 & 0.06 \\
0.75 & 0 & 0.25 \\
0.04 & 0.03 & 0.93
\end{array}\right)
$$

for the $\left(z_{g}, z_{b}\right)$ transition, and

$$
\left(\begin{array}{ccc}
0.99 & 0 & 0.01 \\
0.03 & 0 & 0.97 \\
0 & 0.03 & 0.97
\end{array}\right)
$$

for the $\left(z_{b}, z_{b}\right)$ transition. These matrices imply the kinds of duration numbers mentioned above and that (by definition) long-term unemployment is always preceded by short-term unemployment. We also require that the probability of employment is always higher for the currently employed than for the currently unemployed. ${ }^{6}$

\footnotetext{
6 An exception to one of these restrictions can be found in the transition from the good to the bad aggregate state; there, it is possible to go directly from $\epsilon=e$ to $\epsilon=l$, an outcome necessitated by the requirement that the probability of employment next period be higher for unemployed agents than
} 
Table 1

The distribution of wealth.

\begin{tabular}{|c|c|c|c|c|c|c|c|}
\hline & \multicolumn{5}{|c|}{$\%$ of wealth held by top } & \multirow{2}{*}{$\begin{array}{l}\text { Fraction with } \\
\text { wealth }<0\end{array}$} & \multirow{2}{*}{$\begin{array}{l}\text { Gini } \\
\text { coefficient }\end{array}$} \\
\hline & $1 \%$ & $5 \%$ & $10 \%$ & $20 \%$ & $30 \%$ & & \\
\hline One kind of unemployed & $24 \%$ & $54 \%$ & $72 \%$ & $86 \%$ & $91 \%$ & $10 \%$ & 0.81 \\
\hline Two kinds of unemployed & $26 \%$ & $56 \%$ & $72 \%$ & $83 \%$ & $87 \%$ & $10 \%$ & 0.77 \\
\hline Data & $30 \%$ & $51 \%$ & $64 \%$ & $79 \%$ & $88 \%$ & $11 \%$ & 0.79 \\
\hline
\end{tabular}

This calibration means that the expected duration of unemployment conditional on a bad aggregate state is quite long: 80 periods for long-term unemployed; it is only 2 periods in the good aggregate state. However, note that recessions last only 8 periods on average, so the average duration of an unemployment spell is relatively short. Relatedly, the fraction of all unemployed agents who are long-term unemployed is $73 \%$ in bad times and $33 \%$ in good times, with the total number of the short-term unemployed barely changing at all across aggregate states. Thus, employment dynamics change a lot with the aggregate state and eliminating the risk of unemployment by integrating out the aggregate fluctuations will be a potentially important reduction in unemployment risk; we will get back later to just how much, given this calibration.

We obtain long-run wealth distributions as tabulated in Table $1 .^{7}$ These distributions are roughly consistent with the data in Wolff (1994) and Díaz-Giménez et al. (1997).

\subsection{Model solution: Approximate aggregation}

The models with aggregate uncertainty are solved as in Krusell and Smith (1998). We also compute transition paths for economies without aggregate shocks; here, we postulate a time path for aggregate capital, solve for agents' decisions given this path, and then verify that the time path for aggregate capital implied by agents' aggregated decisions matches the postulated time path. Appendices B and D describe the algorithm in detail.

We obtain equilibrium laws of motion for aggregate capital, which also describe the accuracy of our computations, as follows. For the baseline calibration,

$$
\begin{aligned}
& \log \bar{k}^{\prime}=0.102+0.960 \log \bar{k}, \\
& R^{2}=0.999985, \quad \hat{\sigma}=0.0075 \%
\end{aligned}
$$

in good times (state $z_{g}$ ) and

$$
\begin{aligned}
& \log \bar{k}^{\prime}=0.094+0.961 \log \bar{k}, \\
& R^{2}=0.999946, \quad \hat{\sigma}=0.0141 \%
\end{aligned}
$$

in bad times (state $z_{b}$ ), where $\hat{\sigma}$ is the estimated standard deviation of the error in a regression using simulated time-series data. For the calibration with short- and long-run unemployed, the corresponding equations are

$$
\begin{aligned}
& \log \bar{k}^{\prime}=0.104+0.959 \log \bar{k}, \\
& R^{2}=0.99998, \quad \hat{\sigma}=0.0087 \%
\end{aligned}
$$

in good times (state $z_{g}$ ),

$$
\begin{aligned}
& \log \bar{k}^{\prime}=0.117+0.951 \log \bar{k}, \\
& R^{2}=0.99968, \quad \hat{\sigma}=0.0307 \%
\end{aligned}
$$

in bad times when the last period was bad as well (state $z_{b}$ ), and

\footnotetext{
for employed agents. Because we insist that all newly unemployed receive $\psi_{s}$, this actually requires using a third aggregate state in the formal analysis along with a fourth individual state. Thus, we denote a bad aggregate state following a good aggregate state $z_{d}$ (for "downturn"), to be distinguished from a bad aggregate state following a bad aggregate state (denoted $z_{b}$ ), and we denote the long-term unemployed who directly transited from employment, and therefore receive $\psi_{s}$, by $f$ (for "fired"). By construction, the state $f$ appears only when the aggregate state is $z_{d}$. The implied, larger transition matrices are tabulated in Appendix D.

7 We use the following formula for the Gini coefficient:
}

$$
G=\frac{1}{n}\left(n+1-2\left(\frac{\sum_{i=1}^{n}(n+1-i) k_{i}}{\sum_{i=1}^{n} k_{i}}\right)\right)
$$

for the simulated values of the individual wealth $k_{i}, i=1, \ldots, n$, and $k_{i}$ is ordered in a non-decreasing manner $\left(k_{i} \leqslant k_{i+1}\right)$. Here we use this formula directly to the simulated data although the simulated data contains substantial number of the individuals with negative wealth. 


$$
\begin{aligned}
& \log \bar{k}^{\prime}=0.093+0.963 \log \bar{k}, \\
& R^{2}=0.99998, \quad \hat{\sigma}=0.0077 \%
\end{aligned}
$$

in bad times when the last period was good (state $z_{d}$ ). The fit is a little worse here than in the baseline case, especially when two bad aggregate shocks hit in succession, but it is still impressive.

\section{Welfare effects of eliminating the business cycle}

One of our main objectives is to record the welfare effects of removing cycles for different types of consumers at the time of the removal of cycles, since we expect these effects to vary substantially across different groups of the population. Therefore, we need to solve for transition paths; if we solved only for long-run outcomes, we would be able to comment only on how certain groups do in the long run, and we would not be able to ask the question "How is agent $x$ affected by the removal of cycles?", where $x$ refers to a consumer's type on the date that cycles are (unexpectedly) removed. As in Krusell and Smith (1999), in the transition experiment we therefore replace the stochastic $z$ process with its conditional expectation as of the initial date, leaving a deterministic movement in $z$ and $u$ which disappears in the long run.

The wage income process without aggregate shocks is significantly more complex than the one with aggregate shocks. Using the baseline case as an illustration, recall from the discussion of the one-shot case of aggregate risk in Section 2.3 .2 that the employment process in the economy without cycles has a 3-point support: 0,1 , and a number strictly between 0 and 1 , namely, the conditional probability of a good aggregate state. In the fluctuating economy, a lucky agent with a low realization of the "pure idiosyncratic shock" $i$ is employed no matter what the aggregate state is, and an unlucky consumer with a high realization of $i$ is unemployed no matter what the aggregate state is. Therefore, the application of the integration principle for these consumers is simple-assign the new employment value 1 to the former consumer, and assign the new employment value 0 to the latter consumer. A consumer with an intermediate value of $i$ is employed only if the aggregate state is good. The integration principle assigns the expected value (with respect to the aggregate state) of the employment value in the fluctuating economy as the new employment value for this consumer. Since the underlying employment variable $\epsilon$ in the fluctuating economy takes either 1 or 0 , the expected value $\pi_{g} \cdot 1+\pi_{b} \cdot 0=\pi_{g}$ is assigned as the new employment value, where $\pi_{g}$ is the probability that the aggregate state is good and $\pi_{b}$ is the probability that the aggregate state is bad.

The same logic applies when we adapt this procedure to our infinite-horizon economy. In the economy with cycles, the employment status of a consumer at time $t, \epsilon_{t}$, depends on the histories of idiosyncratic and aggregate shocks up to that time, i.e., on $i^{t} \equiv\left\{i_{1}, \ldots, i_{t}\right\}$ and $z^{t} \equiv\left\{z_{0}, \ldots, z_{t}\right\}$. As in the one-shot case described in Section 2.4 , the $i_{t} s$ are assumed to be i.i.d. and uniformly distributed on $[0,1]$. In the economy without cycles, following the integration principle, we determine the employment value of a consumer with "luck" history $i^{t}$ by calculating the expected value of his employment outcome at time $t$, where the expectation is taken with respect to the set of possible histories $\left\{z^{t}\right\}$ of aggregate shocks. In other words, and again using the (two-state) baseline case as an illustration, we set the consumer's employment value at time $t$ in the economy without cycles equal to his probability of employment in the economy with cycles, given his history of "luck" $i{ }^{t}$. If the consumer is unlucky and the elements of his history $i^{t}$ are all close to 1 , then he is unemployed at time $t$ regardless of the realization of the aggregate history $z^{t}$; by the same token, if the consumer is lucky and the elements of $i^{t}$ are all close to 0 , then he is employed at time $t$ regardless of $z^{t}$. For histories $i^{t}$ with intermediate values, the consumer's employment value in the economy without cycles (i.e., his probability of employment given $i^{t}$ ) depends on the aggregate history $z^{t}$, and in this case we compute the consumer's average employment outcome across different histories $z^{t}$.

It turns out that we can calculate the "averaged" employment values in the economy without cycles by tracking the evolution of two state variables for each consumer. One of these state variables is $P_{g t}$, the probability (in the economy with cycles) that a consumer with history $i^{t}$ is employed at time $t$ and that the aggregate state at time $t$ is good; the other, $P_{b t}$, is the probability (again in the economy with cycles) that a consumer with history $i^{t}$ is employed at time $t$ and that the aggregate state at time $t$ is bad. The new employment value in the economy without cycles is then $P_{g t}+P_{b t}$, or the probability that a consumer with history $i^{t}$ would have been employed at time $t$ in the economy with cycles.

As we move forward in time, the set of possible individual and aggregate histories grows, and consequently the number of possible values that the "averaged" employment value can take on increases too. Indeed, for any subinterval contained in $[0,1]$, there is a positive probability that the employment value in the economy without cycles lies in that interval as time approaches infinity.

Appendices A and B provide further details of the updating procedure in the baseline case. When there are two types of unemployment, the employment process is summarized by a five-dimensional vector. The "integration" procedure is more involved in this case, but the principle remains the same; see Appendix D for complete details.

\subsection{The baseline case: Homogeneous unemployed}

First, we present the results for the case where there is only one type of unemployment. To begin, Table 2 contains summary measures of welfare changes across different groups. We convert the difference between the two expected values into a consumption equivalent in the same way that Lucas did; see Appendix $C$ for the exact formula. 
Table 2

Welfare gains from eliminating business cycles.

\begin{tabular}{|c|c|c|c|c|c|c|c|c|}
\hline \multicolumn{2}{|c|}{ Initial state } & \multirow{2}{*}{$\begin{array}{l}\text { Fraction } \\
\text { gaining }\end{array}$} & \multicolumn{6}{|c|}{ Average utility gains in percentage consumption } \\
\hline $\bar{k}$ & $z$ & & All & $\epsilon=1$ & $\epsilon=0$ & $\beta=$ low & $\beta=$ middle & $\beta=$ high \\
\hline 11.2 & $z_{\mathrm{g}}$ & 0.343 & 0.081 & 0.076 & 0.197 & 0.201 & -0.009 & 0.677 \\
\hline 11.2 & $z_{b}$ & 0.386 & 0.103 & 0.083 & 0.288 & 0.259 & 0.017 & 0.634 \\
\hline 12.3 & $z_{g}$ & 0.329 & 0.083 & 0.081 & 0.136 & 0.225 & -0.001 & 0.617 \\
\hline 12.3 & $z_{b}$ & 0.347 & 0.090 & 0.081 & 0.170 & 0.245 & 0.003 & 0.614 \\
\hline
\end{tabular}

Table 3

Average utility gains by wealth group $\left(\bar{k}=11.2, z=z_{\mathrm{g}}\right)$.

\begin{tabular}{|c|c|c|c|c|c|c|c|c|}
\hline & \multicolumn{8}{|c|}{ Utility gain in percentage consumption } \\
\hline & $<1$ & $1-5$ & $5-25$ & $25-50$ & $50-75$ & $75-95$ & 95-99 & $>99$ \\
\hline All & 0.350 & 0.151 & 0.033 & -0.073 & -0.108 & 0.233 & 1.135 & 1.761 \\
\hline$\epsilon=1$ & 0.212 & 0.127 & 0.027 & -0.075 & -0.109 & 0.232 & 1.134 & 1.763 \\
\hline$\epsilon=0$ & 1.051 & 0.395 & 0.149 & -0.021 & -0.084 & 0.250 & 1.159 & 1.667 \\
\hline
\end{tabular}

Table 4

Utility gains for different types of agents $\left(\bar{k}=11.2, z=z_{g}\right)$.

\begin{tabular}{|c|c|c|c|c|c|c|c|}
\hline \multirow[t]{2}{*}{ Type of agent } & \multicolumn{7}{|c|}{ Wealth percentile } \\
\hline & constr. & 0.005 & 0.05 & 0.5 & 0.95 & 0.995 & 0.999 \\
\hline$\epsilon=1, \beta=$ low & 0.516 & 0.413 & 0.275 & 0.063 & 0.437 & 1.255 & 1.560 \\
\hline$\epsilon=1, \beta=$ middle & 0.221 & 0.134 & 0.021 & -0.109 & 0.690 & 1.552 & 1.857 \\
\hline$\epsilon=1, \beta=$ high & 0.063 & 0.010 & -0.028 & 0.023 & 1.090 & 1.964 & 2.273 \\
\hline$\epsilon=0, \beta=$ low & 3.500 & 1.032 & 0.529 & 0.131 & 0.426 & 1.252 & 1.559 \\
\hline$\epsilon=0, \beta=$ middle & 2.629 & 0.640 & 0.225 & -0.071 & 0.677 & 1.550 & 1.856 \\
\hline$\epsilon=0, \beta=$ high & 1.911 & 0.382 & 0.076 & 0.001 & 1.077 & 1.962 & 2.272 \\
\hline
\end{tabular}

Table 2 contains four transition experiments: starting from two different initial capital stocks (each one is associated with a randomly drawn distribution of assets from the stationary stochastic process for this distribution under aggregate uncertainty) and from two different values of the aggregate shock. The results in the table reveal, first, that the average welfare gain from eliminating cycles is a little more than one magnitude larger than that computed by Lucas for the same period utility function: up to $0.08-0.11 \%$ of consumption from Lucas's $0.008 \%$. Second, the distribution of welfare gains reveals substantial heterogeneity. For example, only a little over a third of the population even realize a gain; the rest lose from eliminating cycles. Summary statistics from the point of view of employment and patience type, as of the point in time when cycles are removed, are also displayed in the table. They reveal that unemployed agents lose on average 2-3 times more from cycles and that, across agents with different time preference rates, the most and least patient gain the most. In contrast, the middle group has a welfare gain that on average is similar to Lucas's number. The largest gainers are actually the most patient group, with numbers over $0.6 \%$, i.e., close to two orders of magnitude larger than Lucas's representative-agent number.

Table 2 does not reveal who the losers from eliminating cycles are. We need a breakdown across wealth groups to find the losers, and the following Table 3 provides this kind of information for one of the four transition experiments. The tables corresponding to each of the other three transition experiments differ only slightly from this one; they are contained in Appendix E.1.

Here, a U-shape across wealth levels appears. We see, in particular, that the consumers in the 25th-75th percentiles in the wealth distribution are significant losers. For example, those in the 50th-75th percentile lose one order of magnitude more in consumption equivalents than Lucas's representative agent gains. Moreover, we see that the losses are larger for the employed than for the unemployed. These agents are not very vulnerable to risk, and they lose particularly from the lower wages that result from lower aggregate savings.

The very richest group gains the most; the top percentile in wealth gains more than $1.5 \%$ in consumption equivalents. Clearly, the gains here derive from the increased interest rate. The poorest, represented as the bottom percentile here, gain between around $0.2 \%$ (the employed) and $1.1 \%$ (the unemployed); for this group, the diminished risk seems to be the reason why the gains are high.

Yet another cut of the heterogeneity in gains is compiled in Table 4, where there is a focus on the groups at the extreme ends in the wealth distribution across different patience and employment statuses. ${ }^{8}$

\footnotetext{
8 Note that not all the combinations of the individual states actually are realized in the simulation, since the wealth holding is endogenous. However, we can think of an individual situated in a specific cell due to an unexpected (positive or negative) wealth transfer.
} 
Table 5

The distribution of wealth

\begin{tabular}{|c|c|c|c|c|c|c|c|}
\hline & \multicolumn{5}{|c|}{ Pct. of wealth held by top } & \multirow{2}{*}{$\begin{array}{l}\text { Pct. with } \\
\text { wealth }<0\end{array}$} & \multirow{2}{*}{$\begin{array}{l}\text { Gini } \\
\text { coefficient }\end{array}$} \\
\hline & $1 \%$ & $5 \%$ & $10 \%$ & $20 \%$ & $30 \%$ & & \\
\hline Cycles & 24 & 54 & 72 & 86 & 91 & 10 & 0.81 \\
\hline No cycles & 25 & 59 & 79 & 95 & 98 & 29 & 0.90 \\
\hline Data & 30 & 51 & 64 & 79 & 88 & 11 & 0.79 \\
\hline
\end{tabular}

Table 6

Welfare gains from eliminating business cycles.

\begin{tabular}{|c|c|c|c|c|c|c|c|c|c|c|}
\hline \multicolumn{2}{|c|}{ Initial state } & \multirow{2}{*}{$\begin{array}{l}\text { Fraction } \\
\text { gaining }\end{array}$} & \multicolumn{8}{|c|}{ Average utility gains in percentage consumption } \\
\hline $\bar{k}$ & $z$ & & All & $\epsilon=e$ & $\epsilon=s$ & $\epsilon=f$ & $\epsilon=l$ & $\beta=$ low & $\beta=$ middle & $\beta=$ high \\
\hline 11.3 & $z_{\mathrm{g}}$ & 1.0 & 0.974 & 0.932 & 1.532 & & 3.005 & 1.708 & 0.597 & 3.309 \\
\hline 11.3 & $z_{d}$ & 1.0 & 1.130 & 0.831 & 0.949 & 3.624 & 6.446 & 2.095 & 0.737 & 3.349 \\
\hline 11.3 & $z_{b}$ & 1.0 & 1.522 & 0.853 & 0.931 & & 10.022 & 2.840 & 1.134 & 3.294 \\
\hline 12.1 & $z_{g}$ & 1.0 & 0.932 & 0.905 & 1.353 & & 2.066 & 1.655 & 0.553 & 3.286 \\
\hline 12.1 & $z_{d}$ & 1.0 & 1.039 & 0.836 & 0.956 & 3.239 & 4.055 & 1.917 & 0.654 & 3.213 \\
\hline 12.1 & $z_{b}$ & 1.0 & 1.078 & 0.835 & 0.912 & & 4.159 & 2.009 & 0.689 & 3.237 \\
\hline
\end{tabular}

The effect on agents who are borrowing-constrained can be seen in this table; if they are impatient and unemployed, the reduction in risk amounts to close to a $4 \%$ increase in utility measured in consumption equivalents. This number is 500 times larger than the number provided by Lucas and it is an answer of sorts to the main query in this paper: are there agents in the population who would really gain a lot from the elimination of cycles? The answer is yes, if we think of $4 \%$ as a large number, and even though it may not be on some level, relative to the numbers we find in the literature, it is a large number!

The table also shows that those in the top one-thousandth of the wealth distribution gain over $2 \%$ if they are patient. Neither the very poorest nor the very richest represent large groups. The poor, especially, need to be at, or very close to, the borrowing constraint in order to gain significantly; just a little bit of wealth goes a long way toward lowering the utility losses from the risk. The gains among the richest fall off somewhat less rapidly; there is a larger group of big winners in this group.

Table 5 shows the long-run wealth distribution in the U.S. data and in the economies with and without cycles. This table shows that inequality increases significantly; the interest rate goes up, making the rich richer, and the lowering of individual risk makes the poor less concerned about holding low levels of assets-they engage in less precautionary savings. In an economy without discount-factor heterogeneity, these effects would not appear nearly as strongly (or at all). The effect of removing uninsurable individual risk in an economy with discount-factor heterogeneity, at least of the persistent kind we consider here, is to move close to the complete-markets outcome, which we know will be rather extreme. It would not be degenerate here since the discount factors do vary stochastically, but close enough that the effects on inequality of eliminating cycles are very large. Consumption inequality also increases in the long run. The consumption Gini coefficient increases from 0.13 to 0.14 , despite the decline in the Gini coefficient for earnings (wage income plus unemployment compensation). The Gini coefficient for income (earnings plus the interest income $r k$ ) increases from 0.32 to 0.35 .

\subsection{The case with short- and long-term unemployment}

In this section, we present the results for the case where we make a distinction between short- and long-term unemployment. Table 6 summarizes the welfare gains for different initial capital stocks and aggregate productivity states. ${ }^{9}$

The average gain is now around 1\%, which is more than 100 times larger than Lucas's number. In contrast to the previous case, everyone gains from eliminating business cycles. In particular, the long-term unemployed realizes a very large gain.

Table 7 breaks down the welfare gains for different wealth levels. We see the U-shape pattern again: very poor and very rich consumers receive the largest gains. The significant size of the very rich's gain indicates that the general equilibrium effect is very large. This is because the precautionary savings are very large in the economy with cycles, due to agents fearing long-term unemployment. To match the realistic wealth distribution (by "forcing" some people to borrow), we assumed more dispersed discount factors; the difference between the smallest and the largest $\beta$ is 0.0112 , while it was 0.0072 in the homogeneous unemployment case. The mean discount factor is also lower here, to prevent people from saving too much. The elimination of the business cycle reduces the risk of long-term unemployment substantially (since the risk was strongly tied to being in a recession), and as a result, aggregate savings drop significantly. In the homogeneous

\footnotetext{
9 Recall that here we make distinction between the $z_{d}$ state (a bad state after a good state) and the $z_{b}$ state (a bad state after a bad state) and that the individual state $f$ exists only when the aggregate state is $z_{d}$.
} 
Table 7

Average utility gains by wealth group $\left(\bar{k}=11.3, z=z_{\mathrm{g}}\right)$.

\begin{tabular}{|c|c|c|c|c|c|c|c|c|}
\hline & \multicolumn{8}{|c|}{ Utility gain in percentage consumption } \\
\hline & $<1$ & $1-5$ & $5-25$ & $25-50$ & $50-75$ & $75-95$ & 95-99 & $>99$ \\
\hline All & 2.312 & 1.499 & 1.108 & 0.439 & 0.366 & 1.120 & 4.362 & 6.965 \\
\hline$\epsilon=e$ & 1.769 & 1.190 & 1.056 & 0.418 & 0.351 & 1.113 & 4.360 & 6.973 \\
\hline$\epsilon=s$ & 4.466 & 2.940 & 2.186 & 0.915 & 0.765 & 1.255 & 4.365 & 6.651 \\
\hline$\epsilon=l$ & 6.523 & 4.553 & 2.824 & 1.278 & 1.063 & 1.450 & 4.494 & 6.638 \\
\hline
\end{tabular}

Table 8

Utility gains for different types of agents $\left(\bar{k}=11.3, z=z_{\mathrm{g}}\right)$.

\begin{tabular}{|c|c|c|c|c|c|c|c|}
\hline \multirow[t]{2}{*}{ Type of agent } & \multicolumn{7}{|c|}{ Wealth percentile } \\
\hline & constr. & 0.005 & 0.05 & 0.5 & 0.95 & 0.995 & 0.999 \\
\hline$\epsilon=e, \beta=$ low & 2.431 & 2.305 & 1.912 & 1.011 & 1.153 & 3.969 & 5.004 \\
\hline$\epsilon=e, \beta=$ middle & 1.417 & 1.311 & 0.987 & 0.363 & 2.023 & 5.475 & 6.553 \\
\hline$\epsilon=e, \beta=$ high & 0.837 & 0.796 & 0.715 & 0.899 & 4.249 & 7.934 & 9.057 \\
\hline$\epsilon=s, \beta=$ low & 5.957 & 5.275 & 3.876 & 1.659 & 1.148 & 3.965 & 5.004 \\
\hline$\epsilon=s, \beta=$ middle & 4.128 & 3.601 & 2.489 & 0.805 & 1.995 & 5.470 & 6.552 \\
\hline$\epsilon=s, \beta=$ high & 2.623 & 2.271 & 1.562 & 1.002 & 4.210 & 7.930 & 9.058 \\
\hline$\epsilon=l, \beta=$ low & 8.775 & 7.696 & 5.331 & 2.104 & 1.137 & 3.961 & 5.003 \\
\hline$\epsilon=l, \beta=$ middle & 6.296 & 5.472 & 3.623 & 1.115 & 1.966 & 5.466 & 6.551 \\
\hline$\epsilon=l, \beta=$ high & 4.086 & 3.521 & 2.257 & 1.068 & 4.174 & 7.925 & 9.057 \\
\hline
\end{tabular}

Table 9

The distribution of wealth.

\begin{tabular}{|c|c|c|c|c|c|c|c|}
\hline & \multicolumn{5}{|c|}{ Pct. of wealth held by top } & \multirow{2}{*}{$\begin{array}{l}\text { Pct. with } \\
\text { wealth }<0\end{array}$} & \multirow{2}{*}{$\begin{array}{l}\text { Gini } \\
\text { coefficient }\end{array}$} \\
\hline & $1 \%$ & $5 \%$ & $10 \%$ & $20 \%$ & $30 \%$ & & \\
\hline Cycles & 26 & 56 & 72 & 83 & 91 & 10 & 0.77 \\
\hline No cycles & 37 & 79 & 98 & 110 & 110 & 77 & 1.06 \\
\hline Data & 30 & 51 & 64 & 79 & 88 & 11 & 0.79 \\
\hline
\end{tabular}

unemployment case, the average capital stock falls from 11.78 to 11.75 , while in the short- and long-term unemployment case, the change is from 11.67 to 11.48 .

Table 8 describes the individual welfare gains. ${ }^{10}$ The largest benefit among the poor agents is realized by the constrained agents in long-term unemployment and with a low discount factor. For them, current consumption is very important, implying that the constraint on borrowing is very costly. Moreover, if the economy falls into a recession, these agents are likely to remain long-term unemployed for a long period of time. This risk is substantially reduced by the elimination of the business cycles. Among the richest agents, the most patient gain significantly, since they foresee a large increase in the interest income in future, when the aggregate savings fall substantially. In this case, very rich agents gain more than very poor agents: the general-equilibrium effect is larger than the risk-reduction effect. This is not the case, however, when business cycles are eliminated at a different point in time: if $\bar{k}=11.3$ and $z=z_{b}$, the gain for a constrained agent with $\epsilon=l$ and $\beta=$ low is $30.3 \%$ (which is the largest gain among all the scenarios) while the gains of the richest are below $9 \%$.

Table 9 compares the long-run wealth distributions before and after the removal of cycles. ${ }^{11}$ The change in the wealth distribution is qualitatively similar to that in the homogeneous unemployment case-the wealth distribution is more dispersed and more people have negative asset holdings. Quantitatively, the change is more extreme here-the majority of agents are at negative asset levels.

\section{Summary and concluding remarks}

This paper revisits the question of how a heterogeneous-agent economy might be affected by the elimination of aggregate fluctuations. Lucas (2003) surveys the previous work on the topic in detail, and a number of papers have been written since, exploring new mechanisms and more complex settings. Some of these departures lead to large effect and others do not; Krebs (2007) looks at the uninsurable earnings risk associated with displacement and argues that the cycle can be very costly for subgroups in the population; Mukoyama and Şahin (2006) analyze a model where workers have different skill levels and find larger costs for the low-skilled; and Schulhofer-Wohl (2008) considers preference heterogeneity, regarding

\footnotetext{
10 Tables for other initial aggregate conditions are available in Appendix E.2.

11 The Gini coefficient is contained in $[0,1]$ when all consumers have positive asset levels. Here, the Gini exceeds one since we use the formula in footnote 7 applied to data that contain agents with negative asset holdings. The percent of wealth held by the top $20 \%$ and top $30 \%$ exceed $100 \%$ because of the existence of negative asset holdings.
} 
attitudes toward risk, and finds very small effects. In this paper, we "go back to basics" and find that, even in a rather standard setting where cyclical idiosyncratic wage risk is abstracted from entirely, when we require the model to match the wealth distribution along the lines of Krusell and Smith (1998), the welfare costs of cycles are rather high, and influence the population quite unevenly.

In particular, we find that the average gains from eliminating cycles are significantly higher in the economy we consider here than those found in Lucas's (1987) representative-agent calculations. In the case of homogeneous unemployment, these gains are still quite low in absolute terms: about $0.1 \%$ of average consumption. However, in the case where we distinguish long- from short-run employment, we obtain much larger gains: about one full percentage point of average consumption. The reason for the much larger effect in the latter case is that, first, long-term unemployment is a major risk-when it hits, it implies poor prospects of re-employment and therefore is a large negative shock to present and future income-and, second, this risk is highly cyclical.

The largest gains from removing cycles are recorded in the poorest, most impatient group: close to $4 \%$ of average consumption for the benchmark calibration. The gain for this group mainly derives from lower risk: the employment process is less risky now since a part of this process derives from the aggregate cycle (as unemployment is higher in recessions); moreover, there is also less wage (and interest) rate risk, since these fluctuate if aggregate productivity does. The very richest also gain substantially: over $2 \%$ in the benchmark calibration. The effect on the richest is due to the increase in the interest rate that comes from a fall in precautionary savings. Precautionary savings, of course, fall due to the lower risk exposure in the absence of cyclical fluctuations. On the other hand, close to $65 \%$ lose from the elimination of cycles. The losing group is the large middle class. This group typically is reasonably well insured because they have some wealth-so they do not benefit much from a reduction in risk-and they are employed. Being employed, but not too wealthy, their main income comes from working, and they lose on net since wages fall with the fall in aggregate savings. All of these comparative (cross-group) effects are present also in the alternative calibration, but they show up more significantly there since (the elimination of) risk is more central when there is long-term unemployment. Also, in this case because risk issues are all the more important, all agents gain from the elimination of aggregate risk.

Finally, we note that the effect on long-run wealth inequality from eliminating aggregate uncertainty is rather drastic in our economy: the Gini coefficient goes up from just under 0.8 to around 0.9 in the benchmark calibration-and to a number exceeding 1 in the alternative calibration. This effect is due to the heterogeneity in discount factors underlying the initial wealth distribution: with less individual uninsured risk, wealth holdings of agents with different time preference rates choose even more divergent asset levels, as the more patient lend even more to the less patient early on, leading relative wealth levels go move apart. In the case with long-term unemployment, more than half of the people hold negative levels of asset in the long run when aggregate risk is no longer present.

\section{References}

Atkeson, A., Phelan, C., 1994. Reconsidering the costs of business cycles with incomplete markets. In: Fischer, S., Rotemberg, J. (Eds.), NBER Macroeconomics Annual 1994, pp. 187-207.

Díaz-Giménez, J., Quadrini, V., Ríos-Rull, J.-V., 1997. Dimensions of inequality: Facts on the U.S. distributions of earnings, income, and wealth. Federal Reserve Bank of Minneapolis Quarterly Review 21, 3-21.

İmrohoroğlu, A., 1989. The cost of business cycles with indivisibilities and liquidity constraints. Journal of Political Economy $97,1364-1383$.

Krebs, T., 2003. Growth and welfare effects of business cycles in economies with idiosyncratic human capital risk. Review of Economic Dynamics 6, 846-868. Krebs, T., 2006. Multi-dimensional risk and the cost of business cycles. Review of Economic Dynamics 9, 640-658.

Krebs, T.G., 2007. Job displacement risk and the cost of business cycles. American Economic Review 97 (3), $664-686$.

Krusell, P., Smith Jr., A.A., 1998. Income and wealth heterogeneity in the macroeconomy. Journal of Political Economy 106, 867-896.

Krusell, P., Smith Jr., A.A., 1999. On the welfare effects of eliminating business cycles. Review of Economic Dynamics 2, $245-272$.

Krusell, P., Smith Jr., A.A., 2002. Revisiting the welfare effects of eliminating business cycles. Manuscript. Yale University.

Lucas Jr., R.E., 1987. Models of Business Cycles. Basil Blackwell, New York.

Lucas Jr., R.E., 2003. Macroeconomic priorities. American Economic Review 93, 1-14.

Mukoyama, T., Şahin, A., 2005. Costs of business cycles for unskilled workers. Working paper 15-2005. CIREQ.

Mukoyama, T., Sahin, A., 2006. Costs of business cycles for unskilled workers. Journal of Monetary Economics 53, $2179-2193$.

Schulhofer-Wohl, S., 2008. Heterogeneous risk preferences and the welfare cost of business cycles. Review of Economic Dynamics 11 (4), $761-780$.

Storesletten, K., Telmer, C., Yaron, A., 2001. The welfare costs of business cycles revisited: Finite lives and cyclical variation in idiosyncratic risk. European Economic Review 45, 1311-1339.

Wolff, E.N., 1994. Trends in household wealth in the United States, 1962-83 and 1983-89. Review of Income and Wealth 40, $143-174$. 